\title{
The power of comparison
}

\author{
Bing Zhang ${ }^{1} \cdot$ Alison R. Mercer $^{2}$
}

Received: 20 June 2015 / Revised: 24 June 2015 / Accepted: 25 June 2015 / Published online: 9 July 2015

(C) Springer-Verlag Berlin Heidelberg 2015

This special issue celebrates excellent examples of invertebrate neuroscience. It has its origins in the Cold Spring Harbor Asia (CSHA) Conferences on invertebrate neurobiology held recently in Suzhou, China, and is designed to provide a 'taster' of some of the exciting works presented at these meetings. There are of course very many aspects of invertebrate neurobiology that have seen remarkable advances in recent years. This issue highlights a small number of interesting examples. The topics are as diverse as they are interesting, and illustrate well the excitement that exists in current invertebrate neuroscience.

For many years, there has been intense interest in the neuronal circuits (central pattern generators, CPGs) that produce rhythmic motor patterns supporting activities such as feeding, walking, swimming, and breathing. Sakurai and Katz discuss the unique perspective that the study of gastropod CPGs brings to this field. One important emerging concept is that different and independently evolved circuits using the same set of neurons can generate the same behavior. Their contribution to this special issue illustrates beautifully the power of a comparative approach. This theme is expanded upon by Ayali and colleagues who discuss recent work on an important model of legged locomotion, the cockroach, Periplaneta americana. In their review, they emphasize that motor system activity is modulated by sensory information and that dynamic interactions between the

Alison R. Mercer

alison.mercer@otago.ac.nz

Bing Zhang

zhangbing@missouri.edu

1 University of Missouri, Columbia, MO 65211-7400, USA

2 University of Otago, Dunedin, New Zealand central nervous system and sensory inputs are essential for coordinated movement.

Studies of central pattern generators have influenced profoundly the way we think about neurons, neuronal networks and their functional properties. It is clear, for example, that neuronal circuits can be reconfigured through the action of neuromodulatory neurons to produce a variety of outputs, and that intrinsic membrane properties of neurons are subject to homeostatic regulation. In this special issue, Marder and colleagues describe the effects of temperature on motor patterns generated by the stomatogastric nervous system of the crab, Cancer borealis, and consider how neuronal networks and the output they generate remain stable in the face of extreme temperature fluctuations.

Temperature is a central focus also of the contribution from $\mathrm{Xu}$ and colleagues who consider the sophisticated mechanisms that have evolved to enable animals to sense and adapt to changes in temperature. In their review, these authors present a strongly comparative viewpoint, and their discussion offers fascinating insights into the effects of temperature on aging and longevity.

Homeostasis, as an overarching theme, is central also to the contribution by Keene and colleagues, who discuss how the brain of the fruit fly modulates sleep and how sleep in this insect, and in other animals, is influenced by metabolic state. Insects have a long history in the study of sleep. Cockroaches, for example, were used to provide the first demonstration that animals show compensatory mechanisms after sleep loss (Campbell and Tobler, Neurosci Biobehav Rev 8:269-300, 1984), and the genetic mechanisms that underlie sleep and sleep disorders are now being investigated extensively using the fruit fly, Drosophila.

Callaerts and colleagues use Drosophila to provide insights into the importance of glia in normal brain function. They explore what is, and what is not, known about 
the contribution of glia to normal behaviors in the fruit fly, including its putative role in regulating circadian rhythmicity, locomotion, sleep, learning and memory and courtship behavior. It is increasingly clear as noted in the contribution by Sivanantharajah and Zhang that understanding behavior requires a better understanding of the connectivity between neurons and glia, and the significance of their functional interactions. Sivanantharajah and Zhang describe state-ofthe-art techniques that have been developed for high-resolution mapping of behavioral circuits in Drosophila.

Kaneko and Ye focus our attention on neural circuits of sensory systems, and how topographic maps are built and refined using mechanisms that are hardwired and experience dependent. By comparing sensory circuits in vertebrates and invertebrates, these authors provide us with an understanding of how circuit wiring is instructed by neural activity.

There is intense current interest in long noncoding RNAs (lncRNAs) and their potential function in biological regulation. What are IncRNAs, and what is known about the biology of these molecules? $\mathrm{Li}$ and Liu provide us with an overview of progress made toward understanding the biological functions of lncRNAs, their mechanisms of action and their contribution to the development and behavior of the fruit fly.

Male courtship behavior in Drosophila takes center stage in the review by Yamamoto and colleagues who explore the critical role played by the fruitless (fru) gene in the formation of sexually dimorphic neural circuitries in Drosophila, and how these circuits operate to guide sex-specific courtship behavior in the fly. The importance of pheromone communication in flies is introduced also in this review, a theme that is expanded upon in an interesting way by Ejima who describes the multiple functions in Drosophila of the male-specific lipid, cis-vaccenyl acetate.

We are treated also to a review of adaptive camouflage in cuttlefish, a behavior that captures the imagination of anyone who watches how quickly and effectively cuttlefish select body patterns that ensure their concealment. In this special issue, Chiao and colleagues review recent research on the visual mechanisms involved.

Last, but by no means least, Hochner and colleagues review their work on the mechanisms of learning-related synaptic plasticity in the cephalopod brain. Their contribution provides a very interesting perspective on the evolution of advanced learning and memory systems.

We are grateful to all of the authors for their contributions, and extend sincere thanks also to Drs. David Stewart and Maoyen Chi, as well as to CSHA, for the support they have given to the Invertebrate Neurobiology conferences. We hope that in addition to stimulating interest, this special issue will motivate and encourage young scientists to become involved in the exciting and rapidly moving field of invertebrate neuroscience. 